\title{
PAPER
}

\section{Visual association test to detect early dementia of the Alzheimer type}

\author{
J Lindeboom, B Schmand, L Tulner, G Walstra, C Jonker
}

J Neurol Neurosurg Psychiatry 2002;73:126-133

See end of article for authors' affiliations

\section{.....................}

Correspondence to: Dr J Lindeboom, Afdeling Medische Psychologie, Medische faculteit VU, kr D345, vd Boechorststraat 7, 1081 BT Amsterdam, The Netherlands; j.lindeboom.psychol@ med.vu.nl

Received 10 September 2001

In revised form

13 February 2002

Accepted

28 February 2002
Background: The visual association test (VAT) is a brief learning task based on imagery mnemonics. The test materials consist of six line drawings of pairs of interacting objects or animals-for example, an ape holding an umbrella. The person is asked to name each object and, later, is presented with one object from the pair and asked to name the other.

Objective: To verify that the task induces robust incidental or effortless learning (study 1), and to study the efficiency of the test as a discriminator between early dementia of the Alzheimer type (DAT) and non-demented people (study 2) and non-DAT types of dementia (study 3).

Methods: Study 1: two groups of elderly volunteers were administered the VAT. The stimuli were presented in the interactive fashion to group A-for example, a monkey carrying an umbrella $(n=83)$ - and side by side to group B-for example, separate pictures of a monkey alone and an umbrella alone $(n=79)$. Group B received learning instructions, but group A did not. Study 2: three groups of subjects were selected from a population based follow up study: incident DAT cases $(n=24)$, cognitively declining subjects not diagnosed with dementia $(n=21)$, and stable non-demented subjects $(n=204)$. Test performance of the non-demented group at baseline was compared with that of patients with DAT at the time of their diagnosis, of patients with DAT a year before their diagnosis, and of nondemented declining subjects at baseline. Study 3: subjects were patients referred for neuropsychological assessment because of suspected dementia. They were diagnosed by consensus criteria of various dementia syndromes.

Results: Study 1: recall was more than twice as high in group A as in group B. Thus interactive presentation, even in the absence of learning instructions, enhances learning. Study 2: at a level of $97.5 \%$ specificity, the VAT had a sensitivity of $87.5 \%$ for DAT cases at the time of diagnosis and $66.7 \%$ one year before diagnosis. The cognitively declining group scored significantly lower on the VAT at baseline than the non-demented group. The VAT discriminated more effectively than both the MMSE and the six item picture learning task from the CAMCOG. Study 3: VAT scores were significantly lower in patients with DAT $(n=48)$ than in patients with vascular dementia $(n=37)$, frontotemporal dementia $(n=9)$, or subcortical dementia $(n=15)$, but not lower than in patients with Lewy body dementia $(n=7)$. Mean mini mental state examination scores of these groups were not significantly different. The VAT discriminated patients with DAT from patients with other types of dementia more effectively than a prose recall test. Sensitivity was $79 \%$ and specificity $69 \%$.

Conclusions: The VAT defects with high specificity a sizeable proportion of patients with DAT a year before the diagnosis, and a low VAT score is relatively uncommon in patients with non-DAT dementia.
M emory impairment is the most pronounced symptom in the early stages of dementia of the Alzheimer type (DAT). ${ }^{1}$ Although this implies that memory tests can detect preclinical DAT with high sensitivity, their specificity would not necessarily be high. A high specificity would require that a low score on the test in question would be characteristic of early DAT but not of other memory deficits occurring in the elderly. As the neuropathology of early DAT is mainly localised in the medial temporal lobe, ${ }^{2}$ its most specific property is the development of increasingly dense anterograde amnesia. The distinctive feature of this memory disorder is that concrete experiences are forgotten in a matter of minutes. Genuine amnesia is rare in the early stages of non-Alzheimer dementias. Therefore, if a test can register signs of true anterograde amnesia, it may detect preclinical DAT with high specificity. We designed such a test, the visual association test (VAT), based on the following rationale.

The primary characteristic of anterograde amnesia is an inability to recall recent day to day experiences. Normally these experiences are recorded without conscious effort. ${ }^{3}$ There are reasons to suppose that the same automatic memory process is involved in visual association learning, also known as imagery mnemonics. ${ }^{4}$ This mnemonic technique consists of forming a mental image of two interacting objects, one representing the item to be remembered and the other a preordained retrieval cue. The formation of the images, in itself, is an effortful activity, but the storage and retrieval of its memory traces probably is not. Firstly, it has been shown that this technique renders the paired associate learning of concrete words virtually effortless, while expanding capacity beyond its usual limits. ${ }^{5}$ Secondly, these effects extend to memory impaired subjects, with the notable and consistent exception of patients with anterograde amnesia. ${ }^{6-9}$

As stated above, the facilitation of paired associate learning is achieved by visualising the items to be remembered in the form of interacting objects. The similarity of this technique to the recall of daily life events seems to reside in the fact that, in both cases, retrieval of the target information is prompted by

Abbreviations: DAT, dementia of the Alzheimer type; VAT, visual association test; MMSE, mini mental state examination; CAMDEX, Cambridge examination for mental disorders of the elderly; GMS, geriatric mental state exam; ROC, receiver operating characteristics; VaD, vascular dementia; FTD, frontotemporal lobar degeneration; DLB, dementia with Lewy bodies 

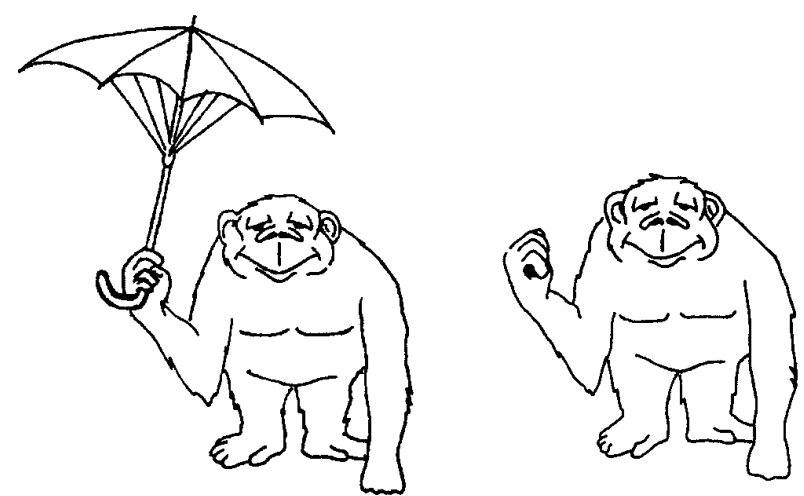

Figure 1 Sample stimulus figure (left) and cue (right) of the visual association test.

intimately related contextual cues. We constructed the VAT with the expectation that the learning process would also be facilitated if the materials were visually presented instead of actively visualised, and if the subject was not explicitly instructed to memorise the materials.

The VAT material consists of six association cards showing two interacting objects (a hedgehog on a chair, an ape holding an umbrella, a key hanging from a balloon, a die in a saucepan, a bird in a baby carriage, and a flag standing in an inkwell) and six cue cards showing only one of the objects. Figure 1 shows a sample item. To ensure that attention is paid, the subject is asked to name the objects on the cue cards-for example, chair, ape-and subsequently to name, in the same order, each pair of interacting objects on the association cards-for example, chair with hedgehog, ape with umbrella. If necessary, naming is aided by the experimenter. Recall is tested without delay, by showing the cue cards again and asking what object is missing-for example, what was the ape carrying? Responses may be oral, written, drawn, or mimed. One point is awarded if the response is sufficiently clear to distinguish the target object from the other objects used in the test. The maximum score is 6 points.

We here present evidence from three separate studies of the VAT. The first is a pilot study showing that the interactive presentation of stimuli in the VAT induces robust incidental learning. The second study concerns a population based sample, and shows that VAT performance distinguishes with high specificity patients with prodromal and early DAT from subjects without dementia. The third study was conducted in a clinical setting, showing that deficient VAT performance is relatively uncommon in patients with non-Alzheimer dementia.

\section{STUDY 1: THE EFFICACY OF INTERACTIVE PRESENTATION}

A pilot study was conducted to verify the expectation that the interactive visual presentation of stimuli in the VAT induces robust incidental learning.
Methods

The subjects were residents of homes for the elderly who volunteered for the study through mediation of the staff. The influence of cognitive deterioration was reduced by excluding subjects who scored below the 10th centile on the cognitieve screening test, ${ }^{10}$ a Dutch adaptation of the short portable mental status questionnaire. ${ }^{11}$ The data for the two conditions (A and B; see below) were collected independently, which led to considerable differences in group composition. However, the groups were comparable in age (table 1). Educational attainment, cognitieve screening test score, and the percentage of women were all higher in group B than in group A.

\section{Procedure}

Group A received the VAT as described above with interacting stimuli and no explicit instructions to memorise the items. In group B, the associations were shown as pictures of two separate objects. The subjects of group $\mathrm{B}$ were instructed to remember that the newly shown (target) object went together with the previously shown (cue) object. Each pair was presented once, but as much time as desired was allowed to rehearse the combinations. In other respects (presentation of cue cards, testing of recall) the procedure was the same for both groups.

\section{Results}

The median number of items recalled was five out of six in group A (interactive presentation), but only two out of six in group B (side by side). The difference is highly significant $(\mathrm{p}<0.0001$, Mann-Whitney U test $)$.

\section{Discussion}

Aside from the manner of presentation, the conditions for recall were more favourable for group B (side by side presentation) than for group A (interactive presentation), because group B was not only given learning instructions and the opportunity to rehearse the items, but was also more educated and obtained higher mental status scores. It is therefore all the more notable that recall was much better in group A. We conclude that the interactive presentation of visual paired associates induces a degree of incidental learning that surpasses intentional learning of the same stimuli when presented in the traditional (side by side) fashion.

\section{STUDY 2: THE VAT IN A COMMUNITY BASED SAMPLE}

The following describes a study on the detection of DAT in a community based sample, comparing the VAT with the mini mental state examination (MMSE) ${ }^{12}$ and another six item visual test of incidental learning.

\section{Methods \\ Subjects}

The subjects were participants of the Amsterdam study of the elderly (AMSTEL), a follow up study on cognitive decline in community dwelling elderly persons aged $65-84$ years. In the

Table 1 Group composition and testing conditions in study 1

\begin{tabular}{llll}
\hline & $\begin{array}{l}\text { Group A } \\
(\mathbf{n}=83)\end{array}$ & $\begin{array}{l}\text { Group B } \\
(\mathbf{n}=78)\end{array}$ & Difference \\
\hline Percentage female & 51 & 82 & $\mathrm{p}<0.0001\left(\chi^{2}\right)$ \\
Age (mean (SD)) & $81.9(6.2)$ & $82.8(5.5)$ & Not significant \\
Education (median (range))* & $3(1-5)$ & $4(2-7)$ & $\mathrm{p}=0.003$ (Mann-Whitney) \\
CST score (mean (SD)) $t$ & $17.0(1.7)$ & $18.5(2.0)$ & $\mathrm{p}<0.001$ ( $t$ test) \\
Stimulus presentation & Interactive & Separated & \\
Learning instructions & No & Yes & \\
\hline
\end{tabular}

*Ordinal scale, range 1 (incomplete primary education) to 7 (university).

†CST, cognitive screening test (Dutch adaptation of SPMSQ), maximum score $=20$. 
Table 2 Group characteristics at baseline (study 2, AMSTEL Project)

\begin{tabular}{|c|c|c|c|}
\hline & $\begin{array}{l}\text { Non-demented } \\
(n=204)\end{array}$ & $\begin{array}{l}\text { Declining } \\
(n=21)\end{array}$ & $\begin{array}{l}\text { Incident DAT } \\
(n=24)\end{array}$ \\
\hline Percentage female & 55 & 71 & 75 \\
\hline Age (mean (SD)) & $72.7(5.2)$ & $77.0(5.0)^{* *}$ & $78.8(4.7)^{* *}$ \\
\hline Education (median (range)) $\dagger$ & $3(1-7)$ & $2(2-6)$ & $2(2-5)$ \\
\hline MMSE (mean (SD)) & $26.9(2.7)$ & $25.6(3.2)^{*}$ & $23.5(3.9)^{\text {* * }}$ \\
\hline \multicolumn{4}{|l|}{ AGECAT depression (\%) } \\
\hline 0. None & 59 & 48 & 58 \\
\hline 1. Slight & 13 & 33 & 13 \\
\hline 2. Mild & 11 & 10 & 17 \\
\hline 3. Moderate neurotic & 11 & 10 & 4 \\
\hline 4. Severe neurotic/psychotic & 4 & 0 & 8 \\
\hline
\end{tabular}

screening phase, a source population of 5666 people was recruited from 30 general practices spread throughout the city. Selection was random except for stratification into four five year age groups. The response rate was $71.5 \%(n=4051)$. All subjects took part on the basis of informed consent. A cohort of 511 was chosen, maintaining age stratification but the sample was selectively weighted to include mostly people with lower MMSE scores. All subjects with MMSE scores below 22 points were included, but only one third of those in the 22-26 range and $6 \%$ of those with a higher score. More details on the design and selection procedure of AMSTEL can be found elsewhere. ${ }^{13}$

\section{Diagnostic procedures and classification}

The cohort members received four yearly diagnostic examinations over a three year period. The follow ups will henceforth be designated as years $1-3$. The examinations included the Cambridge examination for mental disorders of the elderly (CAMDEX), ${ }^{14}$ the geriatric mental state exam (GMS), ${ }^{15}$ and a short medical evaluation. On the basis of these data, the subjects were diagnosed by joint decision of the examining nurse and doctor and the study neurologist (CJ), using DSM-III-R criteria for dementia and NINCDS-ADRDA criteria for probable DAT. For the present purpose, we selected 249 subjects, divided into three groups:

(1) DAT $(n=24)$ : incident cases, diagnosed with DAT in one of the follow ups ( 12 in year 1 and six in years 2 and 3 ).

(2) Declining $(n=21)$ : subjects who were not diagnosed with dementia within the study period, but lost more than 11 points on the CAMCOG (the cognitive section of the CAMDEX) between baseline and year 3. This decline exceeds the $99 \%$ confidence critical change score. ${ }^{16}$

(3) Non-demented $(n=204)$ : subjects who were not diagnosed with dementia on year 3, nor showed cognitive decline as defined above. People with a positive psychiatric or neurological history other than dementia were not excluded.

This selection excluded patients with DAT who were diagnosed at baseline $(n=36)$, cases of non-Alzheimer dementia $(n=10)$, subjects who dropped out of the study without a diagnosis of dementia $(n=207)$, and subjects with missing values on the study variables $(n=9)$. Depression ratings were derived from the GMS with the AGECAT computer program ${ }^{17}$; the symptom levels were converted into an ordinal scale, with psychotic and severe neurotic depression coded as 4. Educational attainment was rated on an ordinal scale ranging from 1 (incomplete primary) to 7 (university); 2 points on this scale indicate a completed primary education, and 4, 5, and 6 points indicate a completed lower, middle, and higher secondary education respectively.

Table 2 shows group characteristics. The non-demented group differed from the other groups in mean age and baseline MMSE score; other differences were not significant.
Neuropsychological tests

Variables used to assess the discrimination between groups were the VAT, the MMSE, CAMCOG picture recall, CAMCOG picture recognition, and age. The VAT scores were independent from the diagnostic procedures, whereas the results from the other measures were incorporated into the diagnostic process. Like the VAT, the CAMCOG picture test measures incidental learning, but reproduction is not cued and is tested after a delay. The subject is asked to name six photographs of objects. Free recall is tested unexpectedly after the administration of seven other CAMCOG items. Next, recognition is tested by choice from photographs depicting three specimens of the object in question.

\section{Statistical analysis}

Firstly, we compared the test variables with regard to distribution, confounding influences, and reliability. Secondly, the discriminative capacities of the tests were evaluated with receiver operating characteristics (ROC) curves. Sensitivity was examined in three subject categories: DAT at the time of diagnosis, the same patients a year before their diagnosis, and declining subjects at baseline. In all three analyses, specificity was based on the baseline scores of non-demented subjects. Age was used to set minimal requirements for discrimination, reasoning that (the cut point of) a test variable is useful only when it has better sensitivity/specificity than a rule assigning all subjects above a certain age to the abnormal group. The ROC curve for age consists of all points classifying subjects above a given age (at the time of examination) as abnormal.

\section{Results}

\section{Psychometric characteristics}

Table 3 lists the frequencies of scores on the memory tasks in non-demented subjects at baseline. Because of the oversampling of subjects with low MMSE scores, the frequencies of low scores are unrepresentative of the whole population. Picture recall is more or less normally distributed and has a floor effect, limiting specificity to a maximum of $96.6 \%$. The distributions of picture recognition and the VAT are highly skewed, with a somewhat more pronounced ceiling effect in the latter. Although dementia was excluded, this group may have contained some cases of amnesia from other causes. The one subject who scored 1 point on the VAT was known to have suffered anoxia during a heart attack, and one subject who scored 2 points had suffered a posterior cerebral artery stroke.

Within the non-demented group, test scores and subject characteristics were unrelated, except for a significant rank correlation between picture recognition and age $(r=-0.25$, $\mathrm{p}<0.01)$, MMSE and education $(r=0.35, \mathrm{p}<0.001)$, and MMSE and depression rating $(r=-0.18, \mathrm{p}<0.05)$.

The reliability of the test variables was measured by the correlation between the first and second administrations 
Table 3 Distribution (in \%) of memory test scores in non-demented subjects ( $n=204)$ at baseline (study 2, AMSTEL Project)

\begin{tabular}{llllllll}
\hline Test score & 0 & 1 & 2 & 3 & 4 & 5 & 6 \\
\hline CAMCOG picture recall & 3.4 & 5.4 & 16.7 & 32.8 & 26.5 & 12.7 & 2.5 \\
CAMCOG picture recognition & 0.5 & 0.5 & 0.5 & 2.0 & 8.3 & 33.3 & 54.9 \\
Visual association test & 0.0 & 0.5 & 2.0 & 6.9 & 7.8 & 15.2 & 67.6 \\
\hline
\end{tabular}

Table 4 Reliability of test scores, measured by the correlation between first (baseline) and second administration (after one year; study 2, AMSTEL Project)

\begin{tabular}{lllll}
\hline & Non-demented & Declining & Incident AD & Total \\
\hline Visual association test & $0.36^{* *}$ & 0.10 & $0.73^{* *}$ & $0.72^{* *}$ \\
CAMCOG picture recall & $0.22^{* *}$ & 0.09 & 0.21 & $0.49^{* *}$ \\
CAMCOG picture recognition & $0.26^{* *}$ & $0.62^{*}$ & 0.36 & $0.59^{* *}$ \\
Mini mental state examination & $0.54^{* *}$ & 0.26 & 0.74 & $0.73^{* *}$ \\
\hline Significance level: * $p<0.01,{ }^{* *} p<0.001$ & & & \\
AD, Alzheimer's disease. & & & \\
\end{tabular}

(interval one year) in the total sample and the three subgroups (table 4). Despite its shortness, the VAT is comparable to the MMSE in overall reliability. In the non-demented group, the low coefficient of the VAT is attributable to restricted variance (the SD was 1.1 points). The low reliabilities in the declining group may be due to subjects deteriorating at varying rates. The internal consistency of the VAT (Cronbach's $\alpha$ ) in the total sample was 0.88 .

\section{Predictive validity}

Figure 2 shows ROC curves for the distinction between test performance of patients with DAT at the time of their diagnosis and non-demented controls at baseline. The VAT discriminated slightly more effectively than the MMSE, reaching optimal results with cut point $2 / 3$ (87.5\% sensitivity, $97.5 \%$ specificity). Picture recognition (not shown) did not discriminate better than age at any level.

In fig 3, sensitivity is based on the scores of the patients with DAT, which were obtained a year before diagnosis. Here, the MMSE is less effective than either the VAT or picture recall, and it hardly discriminates any better than age. Discrimination is optimal with VAT cut points $2 / 3 \quad(66.7 \%$ sensitivity, $97.5 \%$ specificity) and 3/4 (83.3\% sensitivity, $90.7 \%$ specificity). ROC curves for the discrimination of the declining subjects are not shown because it cannot be determined how many of these actually developed DAT. However, it is noted that the difference at baseline between the declining group and the non-demented group was highly significant for the VAT ( $p<0.0001$, Mann-Whitney U test) whereas the effect was weak for the MMSE $(p=0.042)$ and not significant for picture recall and recognition.

\section{Discussion}

The results support our central hypothesis that the VAT detects the memory impairment that is characteristic for DAT. In the diagnosable stage, most patients with DAT score at a level that is rare in persons without dementia. More important, however, is that most DAT cases in this study could already be detected a year before diagnosis with equally high specificity (although with lower sensitivity). Whether prediction is efficient over a longer period remains to be shown. The low average of the declining group suggests that, in at least some cases, the VAT score starts deteriorating more than three years before the diagnosis.

This study was limited by the exclusion of cases of non-Alzheimer dementia. The question whether and to what extent the VAT is failed by such patients is addressed in study 3 .

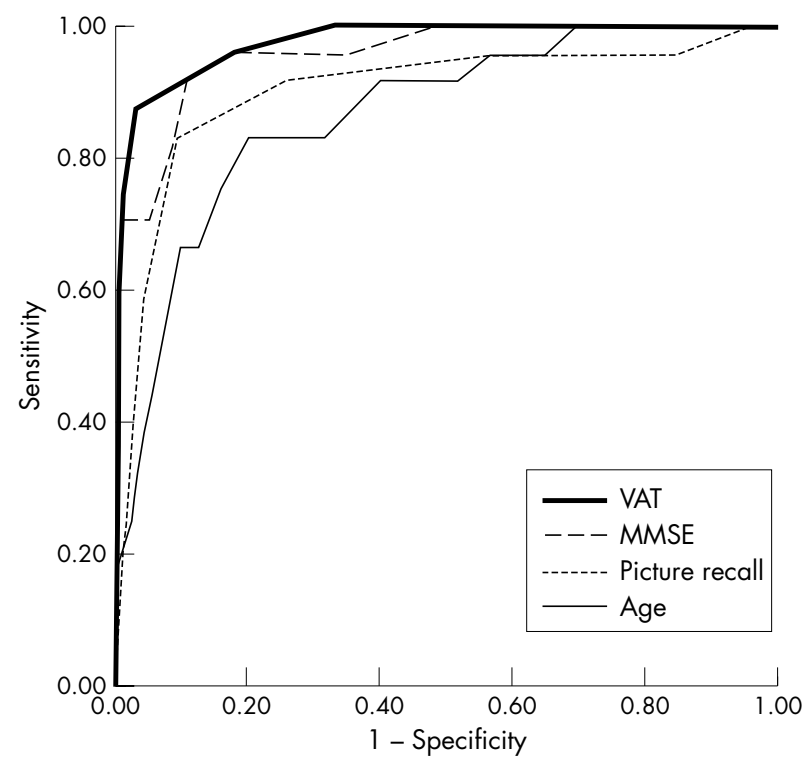

Figure 2 Receiver operating characteristics curves showing discrimination between patients with dementia of the Alzheimer type (at time of diagnosis) and non-demented controls (at baseline) by the visual association test (VAT), CAMCOG picture recall, mini mental state examination (MMSE), and age (study 2, AMSTEL Project)

\section{STUDY 3: THE VAT IN CLINICAL PRACTICE}

In the second study we showed that the VAT is a sensitive and specific indicator of DAT and its prodromal stage. However, the incidence of dementia in the sample of study 2 was low compared with clinical settings, and the few patients who had non-Alzheimer types of dementia were excluded. Therefore, the aim of the third study was to show that a disorder of this kind of incidental learning is also rather specific for DAT with respect to other types of dementia.

\section{Methods}

Subjects

Subjects were patients in the memory clinics and the neurological and geriatric departments of a university hospital and a teaching hospital. All patients were referred for neuropsychological examination because the referring family doctor, geriatrician, or neurologist suspected a dementia syndrome. A total of 224 patients (57.1\% female) were included 


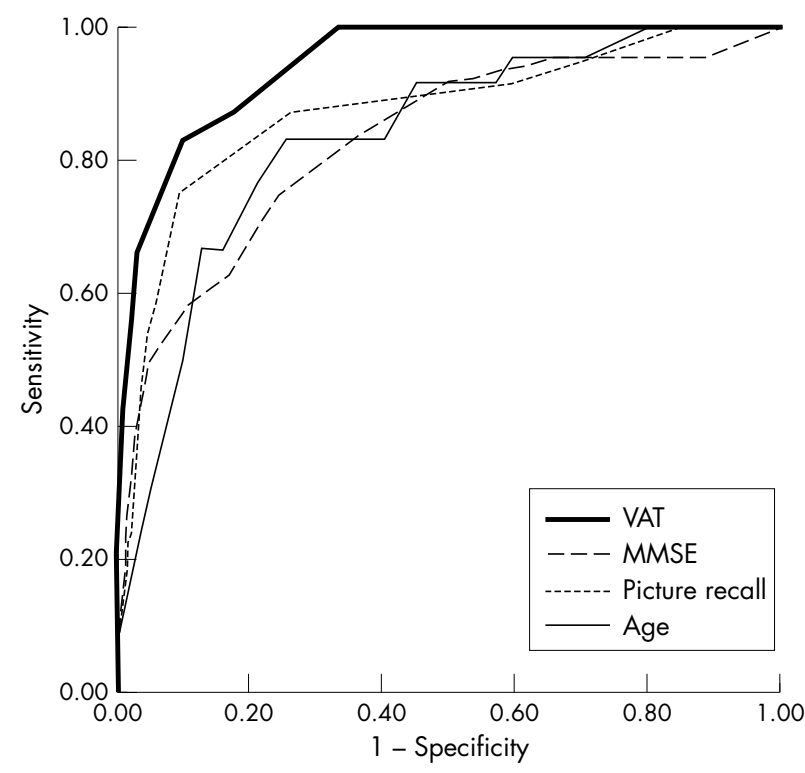

Figure 3 Receiver operating characteristics curves showing discrimination between patients with dementia of the Alzheimer type (one year before diagnosis) and non-demented controls (at baseline) by the visual association test (VAT), CAMCOG picture recall, mini mental state examination (MMSE), and age (study 2, AMSTEL Project).

between September 1996 and March 2000. Their mean age was 76.6 years (SD 7.3; range 51-91), and mean MMSE score was 23.0 (SD 3.8; range 11-30). Median educational level was 4 on a 7 point scale (see legend to table 1), corresponding to about 10 years of full time education; the interquartile range was 2-5 (about 6-11 years of education).

\section{Diagnostic procedures and classification}

All patients were examined at least once by a neurologist or a geriatrician, who carried out the additional laboratory, electroencephalographic, and neuroimaging investigations deemed necessary according to clinical consensus guidelines. ${ }^{18}$ After the history taking, physical and neurological examination, all patients were tested for haemoglobin, thyroid function, renal function, hepatic function, calcium, glucose, syphilis serology, and thiamin, folate, and vitamin B12 deficiency. Other investigations were carried out if indicated. All patients were evaluated by a neuropsychologist (see below). Most cases were discussed in the multidisciplinary staff meetings of the memory clinics.

For the purpose of this study, the clinical diagnoses were checked by strictly applying published consensus criteria on the information available in the patients' files. All clinical inclusion and exclusion criteria of the following five disorders were used:

(1) Alzheimer's disease: DSM-IV criteria and NINCDSARDRA criteria for probable and possible DAT ${ }^{19}$;

(2) vascular dementia (VaD): DSM-IV criteria and NINDSAIREN criteria for probable and possible VaD ${ }^{19}{ }^{21}$;

(3) frontotemporal lobar degeneration (FTD $)^{22}$;

(4) dementia with Lewy bodies (DLB) ${ }^{23}$;

(5) subcortical dementia. ${ }^{24} 25$

These criteria were recorded by a clinical neuropsychologist (BS) from the medical files within a time frame of three months before until one month after the neuropsychological examination. This was supplemented with information from the patient history and behavioural observation sections of the psychological report. Neuropsychological test results were not used for diagnostic classification (except the MMSE score, which was used as one of the NINCDS-ARDRA criteria). If a patient received diagnoses from more than one of these five disease categories, diagnoses of VaD, FTD, or DLB were given priority (in this order) over other diagnoses; a diagnosis of DAT was given priority over a diagnosis of subcortical dementia. Any inconsistencies between the clinical diagnosis and this diagnostic check were resolved by a geriatrician (LT) and a neurologist (GW), who reviewed the cases concerned (except their neuropsychological test results) to decide on the final diagnostic classification. A second file review was necessary in 21 cases; in 15 of these cases the original clinical diagnosis was maintained in favour of the result of the diagnostic check.

\section{Neuropsychological tests}

All patients were evaluated by an experienced neuropsychologist. A flexible approach was used; tests were applied as appropriate in view of the referral question and the characteristics of the patient. However, the following two memory tests were always used. The VAT was administered in the way described above. The prose recall subtest of the Rivermead behavioural memory test was also included in the evaluation. ${ }^{26}{ }^{27}$ In this test, a short, 21 item newspaper article is read to the patient, who is instructed to repeat as many items as possible immediately after presentation and after a median interval of 13 minutes (interquartile range 10-15 minutes). The MMSE score was obtained from the medical files. It was used in the diagnostic classification and as a measure of disease progression.

\section{Statistical analysis}

Firstly, the basic characteristics and MMSE scores of the diagnostic groups were compared by analysis of variance and nonparametric tests. Secondly, the memory test scores of the diagnostic groups were compared by analysis of variance, covarying for disease progression (MMSE). Analyses of variance were followed up with Bonferroni corrected post hoc analyses, accepting group differences with $\mathrm{p}<0.0125$ as significant.

It was expected that the DAT group would score worse on the VAT than the other groups, and that this was not (or to a lesser extent) the case for immediate and delayed prose recall. Finally, a logistic regression analysis was performed with DAT versus non-DAT dementia as the dependent variable and with VAT score and the immediate and delayed prose recall scores as independent variables (method stepwise forward). The hypothesis was that the VAT would discriminate better than immediate or delayed prose recall.

\section{Results}

Of all patients included, $116(51.8 \%)$ were diagnosed according to any of the sets of consensus criteria; the remaining patients either were not given a clinical diagnosis of a dementia syndrome or had insufficient data in their medical records to perform a valid diagnostic check. Thirty nine patients (34\% of 116) received diagnoses from more than one of the disease categories. Table 5 shows the distribution of subjects over the diagnostic categories, their basic characteristics, and MMSE scores.

The groups were not significantly different with respect to basic variables, except sex distribution. There were more men in the FTD and subcortical subgroups than in the other groups. The MMSE scores of the diagnostic groups were not significantly different from each other $(p=0.13)$, indicating that the groups were roughly comparable with respect to disease severity. Table 6 shows the results of the VAT and the prose recall test. As expected, there were significant differences in VAT score between the diagnostic groups. Post hoc comparisons showed that patients with DAT scored significantly worse than those with VaD, FTD, or subcortical dementia. The subgroup differences in immediate and delayed prose recall 
Table 5 Basic characteristics and MMSE scores in the diagnostic groups (study 3, memory clinics)

\begin{tabular}{lllllll}
\hline & $\begin{array}{l}\text { DAT } \\
(n=48)\end{array}$ & $\begin{array}{l}\text { VaD } \\
(n=37)\end{array}$ & $\begin{array}{l}\text { FTD } \\
(n=9)\end{array}$ & $\begin{array}{l}\text { DLB } \\
(n=7)\end{array}$ & $\begin{array}{l}\text { Subcortical } \\
(n=15)\end{array}$ & $p$ Value \\
\hline Percentage female & 67 & 59 & 11 & 57 & 40 & 0.02 \\
Age lyears) & $77.0(7.7)$ & $78.5(5.9)$ & $77.7(5.8)$ & $76.4(8.4)$ & $75.3(9.0)$ & 0.67 \\
Level of education & $4(1-7)$ & $4(1-6)$ & $4(1-6)$ & $3(2-5)$ & $4(2-7)$ & 0.13 \\
MMSE score & $21.6(4.0)$ & $22.3(4.1)$ & $22.4(3.2)$ & $21.0(3.6)$ & $24.6(3.1)$ & 0.13 \\
\hline
\end{tabular}

Values are mean (SD) (age and MMSE score) or median (range) (level of education).

DAT, dementia of the Alzheimer type; MMSE, mini mental state examination; VaD, vascular dementia; FTD, frontotemporal lobar degeneration; DLB, dementia with Lewy bodies.

Table 6 Memory test scores of the diagnostic subgroups (study 3, memory clinics)

\begin{tabular}{|c|c|c|c|c|c|c|}
\hline & DAT & $\mathrm{VaD}$ & FTD & DLB & Subcortical & $\mathrm{p}$ Value \\
\hline Visual association test & $1.5(1.6)$ & $3.0(1.9)$ & $4.0(2.2)$ & $2.7(2.5)$ & $4.6(1.5)$ & $<0.001$ \\
\hline Immediate prose recall & $3.4(1.7)$ & $4.6(2.0)$ & $3.7(1.5)$ & $4.2(1.6)$ & $5.9(2.2)$ & 0.008 \\
\hline Delayed prose recall & $0.9(1.1)$ & $2.4(1.9)$ & $2.6(1.4)$ & $1.9(1.6)$ & $3.5(2.3)$ & $<0.001$ \\
\hline
\end{tabular}

Values are mean (SD).

DAT, dementia of the Alzheimer type; VaD, vascular dementia; FTD, frontotemporal lobar degeneration; DLB, dementia with Lewy bodies.

were also significant, but these differences were less pronounced. On immediate prose recall, patients with DAT scored significantly lower than those with subcortical dementia; on delayed prose recall they scored significantly lower than patients with VaD or subcortical dementia $(\mathrm{p}<0.05$ Bonferroni corrected). In the logistic regression analysis, the VAT score was selected first $(p=0.004)$ and the delayed prose recall score second $(p=0.02)$; immediate prose recall was not included in the model $(p=0.89)$. The analysis correctly classified $73 \%$ of DAT cases and $75 \%$ of non-DAT cases. Some $90 \%$ of patients with DAT scored 3 points or less (sensitivity), whereas $50 \%$ of those with non-DAT dementia scored 4 points or more (specificity). Sensitivity and specificity were $79 \%$ and $69 \%$ respectively for VAT scores of 2 or less, and $63 \%$ and $80 \%$ for VAT scores of 1 point.

\section{Discussion}

The sensitivity for DAT of VAT scores below 3 points found in this study $(79 \%)$ was similar to that in study $2(87.5 \%)$. The specificity of this cut point for the distinction between DAT and other types of dementia was $69 \%$.

To prevent circularity in the study, we ignored the results of the neuropsychological evaluation when composing the diagnostic groups, but this may have led to classification errors. Other classification errors may have arisen from overlap between criteria sets, many patients satisfying more than one set of criteria. This overlap is a notorious problem. ${ }^{28}$ We had to make somewhat arbitrary decisions to solve this problem. We gave preference to diagnoses of VaD, FTD, and DLB above DAT, because DAT is essentially a diagnosis by exclusion. Consequently, we reasoned that, if there are arguments in favour of VaD, FTD, or DLB, then they should have preference over DAT. Moreover, possible mixed pathology was ignored, notwithstanding the fact that this is common. ${ }^{29}{ }^{30}$ However, our strategy resulted in a relatively pure DAT group. The diagnostic check differed from the clinical diagnosis of DAT in only two cases (which had to be reclassified after revision). As our main goal was to investigate whether the VAT is able to specifically discriminate DAT from other types of dementia, some "pollution" of the other diagnostic groups was of minor importance. Moreover, the decision procedure ensured, on the one hand, that the results were not biased in favour of the DAT group and, on the other hand, maximised the number of patients in the non-DAT groups, which was regrettably small in some cases. Nevertheless, the "signal" in our findings remained clearly visible against the diagnostic "noise". Our diagnostic groups were comparable in age, educational level, and dementia severity as expressed by the MMSE scores. Therefore differences in VAT scores cannot be ascribed to these potentially confounding influences. It may be claimed that the MMSE is a rather blunt instrument, and that equal MMSE scores do not completely rule out the possibility that the patients with DAT scored lower on the VAT because their dementia was more severe. However, the MMSE is not very sensitive to the more prominent symptoms of non-Alzheimer dementia, such as slowing and executive disorders. ${ }^{30}$ Therefore, given equal MMSE scores, the disease is likely to be more advanced in non-Alzheimer patients than in patients with DAT.

The DLB group contained more patients with a low VAT score, and the mean VAT scores of patients with DLB were not significantly different from those of patients with DAT. Thus, we could not confirm the specificity of the VAT with respect to the distinction between DAT and DLB. This was an unexpected finding because a clear memory disorder is not an obligatory criterion for DLB. In fact, memory function in this disorder has been reported to be only mildly disturbed. ${ }^{31}{ }^{32}$ An explanation for our finding may be that the clinical distinction between DAT and DLB is particularly difficult. ${ }^{33}{ }^{34}$ In particular, the criterion of fluctuations in attention and alertness may be hard to establish in a reliable way. ${ }^{35}{ }^{36}$ This may have caused more misclassifications in the DLB group than in the other groups.

\section{GENERAL DISCUSSION}

The design of the VAT was based on the finding that the use of imagery mnemonics leads to a remarkable improvement in paired associate learning. ${ }^{5}$ As study 1 shows, this effect can be obtained in the absence of active visualisation or a learning intention, the only requirement being that the target and cue are perceived as interacting objects. Imagery mnemonics are inefficient in patients whose memory impairment has amnesic qualities, as in DAT. We therefore expected that patients with DAT would fail on the VAT. This is confirmed by studies 2 and 3. Study 2 shows that low VAT scores distinguish patients with DAT from normals, even in the preclinical phase. In addition, study 3 shows that the test distinguishes DAT from other types of dementia. We found similar sensitivities for DAT in both studies - that is, around $80 \%$ for VAT scores of 2 points or less. The specificity of this cut point for the distinction between early DAT and normalcy was $97.5 \%$ (study 2), 
whereas it was $69 \%$ for the distinction between DAT and other types of dementia (study 3 ). This difference in specificity is to be expected in view of the comparison groups used (normal elderly subjects and patients with non-DAT dementia respectively). We conclude from these findings that the VAT may offer a considerable amount of differential diagnostic information.

The design of the VAT was inspired by the spectacular learning effect of imagery mnemonics. Our results are in accordance with Crovitz's principle of encoding specificity, which holds that recall can be raised to the level of recognition if a cue is encoded along with the target at the time of presentation. ${ }^{37}$ We suppose that the experiences of daily life are encoded and reproduced in similar ways, and that the integrity of these processes is reflected by the incidental learning of visual associations as measured with the VAT. If the VAT indeed reflects the integrity of everyday episodic memory, it will also be a good predictor of practical impairment.

The favourable characteristics of the VAT are probably also due to the fact that paired associate learning taxes hippocampal functions. ${ }^{38}$ It is well known that the hippocampus is one of the first brain structures to deteriorate in DAT. ${ }^{39}$ This explains the sensitivity of the VAT for early DAT. On the other hand, people whose memory functions are intact or relatively mildly deteriorated perform at or near the ceiling of the VAT. The memory problems in non-DAT types of dementia are often not caused by dramatic hippocampal failure but by neuropathology elsewhere in the brain. This probably leaves automatic memory processes relatively undisturbed in nonDAT dementia, but causes disorders in the effortful processes of learning and retrieval. The VAT seems to circumvent these effortful processes, which may explain its specificity.

The validity of the VAT compares favourably to recently published brief screening tests with respect to the distinction between DAT and normalcy, ${ }^{40}{ }^{41}$ as well as with respect to the distinction between DAT and other types of dementia. ${ }^{42}$ However, the briefest of these tests was not investigated in a nonDAT sample, whereas the one that was investigated in non-DAT patients takes more time to administer than the VAT. At first sight, these tests appear to differ from the VAT by showing that delayed recall of patients with DAT is impaired relative to immediate recall. However, it seems probable that immediate recall in these tests is derived from working memory. By erasing the material from working memory, the delay (or the distraction provided) shows that it is not encoded in long term memory. In the VAT, recall is delayed to the extent that the presentation of each item is followed by the presentation or recall of the other five items. Our findings do not offer conclusive proof for an encoding deficit in DAT, but our clinical experience with the VAT supports the possibility. The errors of patients with DAT usually indicate a total unawareness of the targets and often consist of obvious associates of the cue, such as ape-banana. On the other hand, if a patient with DAT succeeds in producing any correct responses, these are likely to be replicated even when recall is tested 30 minutes later. This points to an encoding deficit rather than accelerated forgetting.

In addition to its predictive validity, the VAT has several attractive properties as a screening test. It is not taxing, it takes very little time to administer, and it is not confounded by age, education, or depression. The only prerequisite is that the stimuli can be consciously perceived, which may not be satisfied by patients with severe attention disorders or visual impairment. Of course, we do not imply that administration of the VAT as a stand alone test is sufficient to diagnose DAT. Our study shows that the VAT may be helpful in the early diagnosis of DAT and in the differential diagnosis of dementia syndromes in general. However, more medical and neuropsychological information is needed for a reliable (differential) diagnosis. The diagnostic contribution of neuropsychology is probably greatest in cases that present with cognitive abnormalities in the absence of other neurological or somatic symptoms. In such cases one needs a more refined neuropsychological profile than offered by a short screening test. If, for example, in addition to memory tests such as the VAT we also consider tests of psychomotor speed, executive functioning, and semantic memory, then DAT and other types of dementia may be distinguished with greater precision. ${ }^{43} 44$

In conclusion, the VAT is a promising tool for the assessment of amnesia. Besides the detection of DAT it may be useful for various other purposes, such as diagnosing amnesic syndromes with various causes, monitoring recovery from post-traumatic amnesia, and screening patients eligible for imagery mnemonics training. As the VAT was designed to identify a form of memory impairment that is not readily compensated for, it may also be a good indicator of practical impairment.

\section{ACKNOWLEDGEMENTS}

L Stokhof and M Janse assisted in the data collection for the pilot study. R Heijt and H Smeding assisted in collecting neuropsychological test data for study 3. Professor W A van Gool and E Vissers commented on drafts of the paper. The geriatricians and neurologists of the Slotervaartziekenhuis and Academic Medical Center allowed us to access their patient files. Dr P Green assisted in preparing the manuscript.

\section{Authors' affiliations}

J Lindeboom, Department of Medical Psychology, Vrije Universiteit Medical Centre, Amsterdam, The Netherlands

B Schmand, G Walstra, Department of Neurology, Academisch

Medisch Centrum, Universiteit van Amsterdam, Amsterdam

L Tulner, Geriatric Department, Slotervaart Hospital, Amsterdam C Jonker, AMSTEL Project, Institute of Extramural Research, Vrije Universiteit, Amsterdam

\section{REFERENCES}

1 Welsh KA, Butters N, Hughes JP, et al. Detection and staging of dementia in Alzheimer's disease. Use of the neuropsychological measures developed for the Consortium to Establish a Registry for Alzheimer's Disease. Arch Neurol 1992;49:448-52.

2 Geula C. Abnormalities of neural circuitry in Alzheimer's disease: hippocampus and cortical cholinergic innervation. Neurology 1998;51:S18-29.

3 Hasher L, Zacks RT. Automatic and efforfful processes in memory. J Exp Psychol Gen 1979;108:356-68.

4 Patten BM. The ancient art of memory. Usefulness in treatment. Arch Neurol 1972;26:25-31.

5 Bower GM. Mental imagery and associative learning. In: Gregg L, ed. Cognition in learning and memory. New York: Wiley, 1972.

6 Baddeley AD, Warrington EK. Memory coding and amnesia. Neuropsychologia 1973;11:159-65.

7 Cermak LS. Imagery as an aid to retrieval for Korsakoff patients. Cortex $1975 ; 11: 163-9$

8 Gasparrini B, Satz P. A treatment for memory problems in left hemisphere CVA patients. Neuropsychology 1979:2:137-50.

9 Jones MK. Imagery as a mnemonic aid after left temporal lobectomy: contrast between material-specific and generalized memory disorders. Neuropsychologia 1974;12:21-30.

10 de Graaf A, Deelman BG. Cognitieve screening test. Lisse, The Netherlands: Swets and Zeitlinger, 1991

11 Pfeiffer E. A short portable mental status questionnaire for the assessment of organic brain deficit in elderly patients. J Am Geriatr Soc 1975;23:433-41

12 Folstein MF, Folstein SE, McHugh PR. "Mini-mental state". A practical method for grading the cognitive state of patients for the clinician. $J$ Psychiatr Res 1975;12:189-98.

13 Launer LJ, Dinkgreve MA, Jonker C, et al. Are age and education independent correlates of the Mini-Mental State Exam performance of community-dwelling elderly? J Gerontol 1993;48:271-7.

14 Roth M, Tym E, Mountioy CQ, et al. CAMDEX. A standardised instrument for the diagnosis of mental disorder in the elderly with special reference to the early detection of dementia. Br J Psychiatry 1986;149:698-709.

15 Copeland JR, Kelleher M, Kellett JM, et al. A semi-structured clinical interview for the assessment of diagnosis and mental state in the elderly: the Geriatric Mental State Schedule. I. Development and reliability. Psychol Med 1976;6:439-49.

16 Lindeboom J, Ter Horst R, Hooyer C, et al. Some psychometric properties of the CAMCOG. Psychol Med 1993;23:213-19.

17 Copeland JR, Dewey ME, Griffiths-Jones HM. A computerized psychiatric diagnostic system and case nomenclature for elderly subjects: GMS and AGECAT. Psychol Med 1986;16:89-99. 
18 CBO. Centraal Begeleidingsorgaan voor Intercollegiale Toetsing. Herziening Consensus Diagnostiek bij het Dementiesyndroom. Utrecht, The Netherlands: CBO, 1997.

19 American Psychiatric Association. Diagnostic and statistical manual of mental disorders; DSM-IV. Washington DC: APA, 1994.

20 McKhann G, Drachman D, Folstein M, et al. Clinical diagnosis of Alzheimer's disease: report of the NINCDS-ADRDA Work Group under the auspices of Department of Health and Human Services Task Force on Alzheimer's Disease. Neurology 1984;34:939-44.

21 Roman GC, Tatemichi TK, Erkinjuntti T, et al Vascular dementia: diagnostic criteria for research studies. Report of the NINDS-AIREN International Workshop. Neurology 1993;43:250-60.

22 Neary D, Snowden JS, Gustafson L, et al. Frontotemporal lobar degeneration: a consensus on clinical diagnostic criteria. Neurology 1998:51:1546-54.

23 McKeith IG, Galasko D, Kosaka K, et al. Consensus guidelines for the clinical and pathologic diagnosis of dementia with Lewy bodies (DLB): report of the consortium on DLB international workshop. Neurology 1996;47:1113-24.

24 Brown RG, Marsden CD. 'Subcortical dementia': the neuropsychological evidence. Neuroscience 1988;25:363-87.

25 Cummings JL, Benson DF. Subcortical dementia. Review of an emerging concept. Arch Neurol 1984;41:874-9.

26 Van Balen HGG, Wimmers MFGH. Rivermead behavioural memory test. Normeringsgegevens voor Nederland en Vlaanderen. Lisse, The Netherlands: Swets and Zeitlinger, 1993.

27 Wilson B, Cockburn J, Baddeley A. The Rivermead behavioural memory test. Suffolk: Thames Valley Test Company, 1991.

28 Erkinjuntti T, Ostbye T, Steenhuis R, et al. The effect of different diagnostic criteria on the prevalence of dementia. N Engl J Med 1997;337: 1667-74

29 Bowler JV, Munoz DG, Merskey H, et al. Fallacies in the pathological confirmation of the diagnosis of Alzheimer's disease. J Neurol Neurosurg Psychiatry 1998;64:18-24.

30 Rothlind JC, Brandt J. A brief assessment of frontal and subcortical functions in dementia. J Neuropsychiatry Clin Neurosci 1993;5:73-7.

31 Holmes C, Cairns N, Lantos P, et al. Validity of current clinical criteria for Alzheimer's disease, vascular dementia and dementia with Lewy bodies. Br J Psychiatry 1999;174:45-50.
32 Ballard CG, Ayre G, O'Brien J, et al. Simple standardised neuropsychological assessments aid in the differential diagnosis of dementia with Lewy bodies from Alzheimer's disease and vascula dementia. Dement Geriatr Cogn Disord 1999;10:104-8.

33 Heyman A, Fillenbaum GG, Gearing M, et al. Comparison of Lewy body variant of Alzheimer's disease with pure Alzheimer's disease: Consortium to Establish a Registry for Alzheimer's Disease, Part XIX. Neurology 1999;52:1839-44.

34 Gomez-Tortosa E, Newell K, Irizarry MC, et al. Clinical and quantitative pathologic correlates of dementia with Lewy bodies. Neurology 1999;53:1284-91.

35 Verghese J, Crystal HA, Dickson DW, et al. Validity of clinical criteria for the diagnosis of dementia with Lewy bodies. Neurology 1999;53:1974-82

36 McKeith IG, Ballard CG, Perry RH, et al. Prospective validation of consensus criteria for the diagnosis of dementia with Lewy bodies. Neurology 2000:54:1050-8.

37 Tulving $\mathbf{E}$, Thomson DM. Encoding specificity and retrieval processes in episodic memory. Psychol Rev 1973;80:352-73

38 Rombouts SA, Machielsen WC, Witter MP, et al. Visual association encoding activates the medial temporal lobe: a functional magnetic resonance imaging study. Hippocampus 1997;7:594-601.

39 Fleischman DA, Gabrieli J. Long-term memory in Alzheimer's disease. Curr Opin Neurobiol 1999;9:240-4.

40 Buschke H, Kuslansky G, Katz M, et al. Screening for dementia with the memory impairment screen. Neurology 1999;52:231-8.

41 Solomon PR, Hirschoff A, Kelly B, et al. A 7 minute neurocognitive screening battery highly sensitive to Alzheimer's disease. Arch Neurol 1998;55:349-55.

42 Mathuranath PS, Nestor PJ, Berrios GE, et al. A brief cognitive test battery to differentiate Alzheimer's disease and frontotemporal dementia. Neurology 2000;55:1613-20.

43 Perry RJ, Hodges JR. Differentiating frontal and temporal variant frontotemporal dementia from Alzheimer's disease. Neurology 2000:54:2277-84

44 Gainotti G, Marra C, Villa G. A double dissociation between accuracy and time of execution on attentional tasks in Alzheimer's disease and multi-infarct dementia. Brain 2001;124:731-8.

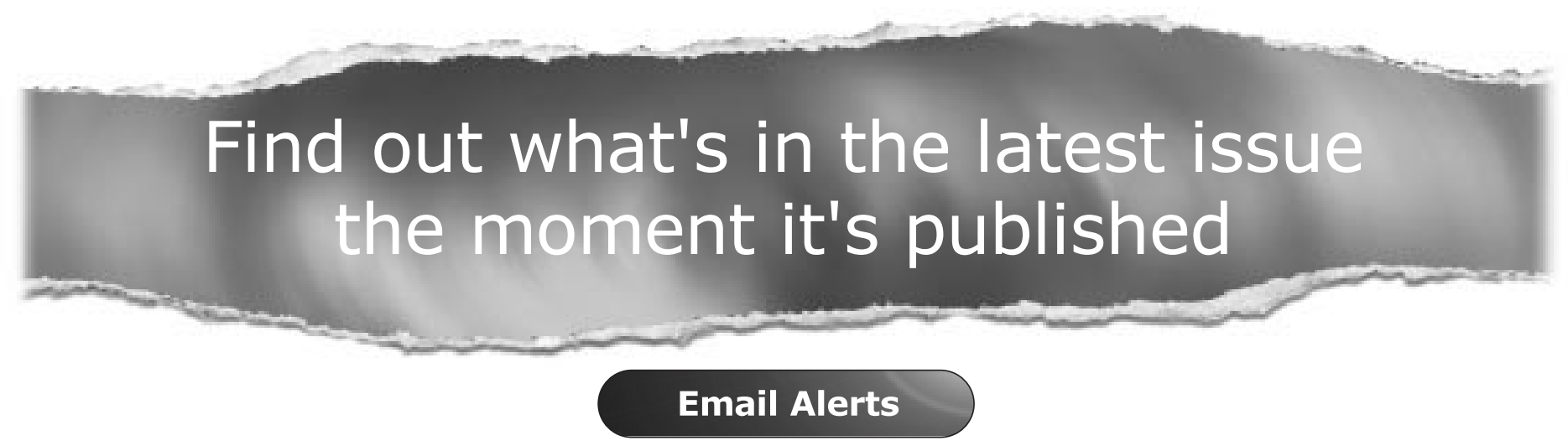

Sign up to receive the table of contents by email every month. You can select from three alerts: Table of Contents (full), TOC Awareness (notice only); Journal of Neurology, Neurosurgery, and Psychiatry related announcements.

www.jnnp.com 